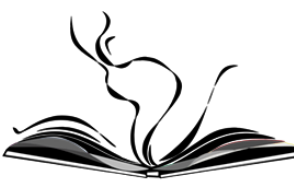

Wagner Iglecias ${ }^{7}$

Universidade de São Paulo, Brasil Ana Esther Ceceña ${ }^{2}$ (iD) Universidad Nacional Autónoma de México, México

Versión en español English version

\title{
Carta às leitoras e aos leitores,
}

A Brazilian Journal of Latin American Studies (BJLAS) tem o prazer de publicar a edição de número 37 da revista especializada em estudos sobre a América Latina e o Caribe. Nesta oportunidade apresentamos o dossiê "Relações China - América Latina e Caribe", no qual especialistas fazem análises sobre tão ampla temática a partir de distintos pontos de vista. O pano de fundo das discussões são as transformações ocorridas nas relações globais de economia e poder nas últimas décadas. A antiga ordem mundial do Pós-Guerra - liderada pelos Estados Unidos em parceria com a Europa Ocidental e o Japão, e sustentada pela arquitetura de governança global composta pela Organização das Nações Unidas, pelo Fundo Monetário Internacional e pelo Banco Mundial - já não reflete a conjuntura internacional contemporânea. Novos atores entraram em cena neste início de século. A Índia tem emergido como potência econômica, a Rússia recobrou importância na arena política mundial após a débâcle soviética e a China, maior império da Antiguidade, encontra-se em acelerado movimento de (re)ascensão ao topo do poder mundial.

\footnotetext{
${ }^{1}$ Doutor em Sociologia pela Universidade de São Paulo. É professor do Curso de Gestão de Políticas Públicas da Escola de Artes, Ciências e Humanidades e do Programa de Pós-Graduação em Integração da América Latina da Universidade de São Paulo. E-mail: wi6@usp.br

2 Doutora em Relações Econômicas Internacionais pela Université de Paris I, Sorbonne. Pesquisadora do Instituto de Investigaciones Económicas e Postgrado em Estudios Latinoamericanos da Universidad Nacional Autónoma de México. E-mail: anacecena@gmail.com
}

Brazilian Journal of Latin American Studies - Cadernos Prolam/USP, v. 19, n. 37, p. 01-21, out. 2020. 
A América Latina e o Caribe, embora sem protagonismo global, parecem ser uma região fundamental neste período de transição entre a breve ordem unipolar estado-unidense, que vigorou entre o fim da ex-URSS e o início do século XXI, e a nova ordem multipolar que está sendo construída. Enquanto os Estados Unidos veem a América Latina e o Caribe, desde o início do século XIX, como uma área natural de sua expansão econômica, geopolítica e cultural, a China tem na região um provedor de commodities agrícolas e energéticas fundamentais à sua economia. $\bigcirc$ aprofundamento de laços econômicos de Beijing com nossos países, através do aumento do comércio bilateral, dos investimentos e dos empréstimos, tem sido um dos principais motivos do aumento das tensões entre Estados Unidos e China. As relações econômicas com a potência asiática, se por um lado nos proporcionam acesso para a exportação de nossos produtos a um mercado gigantesco, garantindo maior grau de autonomia aos nossos países nas relações com os tradicionais centros de poder mundial, por outro contribuem para a manutenção do nosso perfil primário-exportador. Trata-se, como se vê, de relações plenas de complexidade, e refletir sobre o lugar da nossa região nessa disputa global por hegemonia é tarefa fundamental para todas e todos que se dispõem a pensar a América Latina e o Caribe.

Este dossiê começa com o artigo "DA GRANDE CRISE À GRANDE DEPRESSÃO DOS 2020's. DESENVOLVIMENTO DA CHINA, REFLEXÃO PÓS-KEYNESIANA E PERSPECTIVAS", de Alicia Girón, da Universidad Nacional Autónoma de México, México. Nele a autora discute a crise econômica mundial de 2020, provocada pela pandemia da Covid-19, suas consequências sobre a economia chinesa. A autora assinala os graves antecedentes desta crise presentes nas medidas econômicas ortodoxas implementadas em vários países do mundo por conta da crise de 2008, com fortes impactos sobre a atividade produtiva e o nível de emprego. No entanto, ela assinala também que o Banco Central chinês apostou, na mesma época, numa política econômica expansiva, conforme já vinha 
fazendo desde a entrada do país na Organização Mundial do Comércio (OMC), em 2001. Mais que isso, Beijing investiu, a partir de 2013, num modelo de desenvolvimento voltado ao exterior, a Nova Rota da Seda, envolvendo Ásia, África e América Latina através de circuitos terrestres e marítimos destinados a integrar fornecedores e consumidores de produtos vinculados à economia chinesa. Rico em dados quantitativos o artigo aponta para possíveis consequências, nestas parcerias, resultantes da desaceleração atual das economias dos países envolvidos.

O segundo artigo é de autoria de Gabriel Merino, da Universidad Nacional de La Plata, Argentina, intitulado "A ASCENSÃO DA CHINA E AS DISPUTAS ESTRATÉGICAS NOS GRUPOS DOMINANTES DOS ESTADOS UNIDOS". Nele o autor presenteia os leitores com uma profunda discussão sobre as grandes transformações da economia e da geopolítica contemporâneas envolvendo Estados Unidos e China. O foco de Merino são as diversas estratégias desenvolvidas por Washington para fazer frente ao crescimento chinês e à consequente disputa pela hegemonia global. Ao mesmo tempo o artigo reflete sobre as respostas de Beijing diante do giro nacionalista-americanista representado pela chegada de Donald Trump ao poder. Merino sustenta que as iniciativas da Casa Branca desde então têm ampliado a crise da ordem mundial e podem, por conta disto, favorecer indiretamente a China. Enquanto as forças globalistas debilitam-se no Ocidente a China aproveita a oportunidade para sua influência estratégica em direção a seu entorno próximo, na Ásia, e também em direção à África e à América Latina.

\section{Em "O POUSO DO DRAGÃO NA AMÉRICA DO SUL: UMA ANÁLISE} DA GEOECONOMIA, DOS CHINA'S POLICY PAPER ON LATIN AMERICA AND THE CARIBBEAN E DA NOVA ROTA DA SEDA" Bernardo Rodrigues, da Universidade Federal do Rio de Janeiro, Brasil, apresenta dados importantes sobre a crescente incorporação da América do Sul na estratégia de expansão geoeconômica global da China. Analisando o período 2001-2016 a partir de dados quantitativos e dos policy papers 
chineses para a região, o autor demonstra como Beijing tem na América Latina e no Caribe uma prioridade em direção à construção de uma nova ordem mundial. Esta seria pautada pelo multilateralismo, pela cooperação entre as nações e pela integração dos países do Sul às cadeias globais de valor. Para tanto, sustenta o artigo, é fundamental que as lideranças políticas sul-americanas interajam com Beijing de maneira autônoma, visando garantir acesso a tecnologias e conhecimento que permitam o estabelecimento de relações de independência simétrica da região em relação à potência asiática.

Lourdes Regueiro Bello, do Centro de Investigaciones de Política Internacional, de Cuba, discute, em "CENTROAMÉRICA NA DISPUTA GEOPOLÍTICA ENTRE A CHINA E OS ESTADOS UNIDOS" a posição da América Central na disputa comercial global travada entre Estados Unidos e China. Como se sabe este enfrentamento vai além da economia, e tem também implicações geopolíticas. E a América Central, por sua condição ístmica e bioceânica, possui papel estratégico nesta disputa. Washington considera a presença chinesa numa região tão próxima a seu território como uma ameaça ao mesmo tempo em que exerce posição similar em relação a Taiwan e a grande parte do Mar da China Meridional. A percepção de ameaça vai além, por conta do convite feito por Beijing aos países da América Latina para fazer parte da já mencionada Nova Rota da Seda. No caso específico das nações centro-americanas a oferta de recursos econômicos feita por Beijing é, por um lado, tentadora e quase irrecusável, mas por outro pode implicar em duras sanções por parte de Washington. O artigo de Regueiro nos ajuda a especular sobre os próximos capítulos deste interessante xadrez.

Na sequência apresentamos o artigo "RELAÇÕES ENTRE A CHINA E AMÉRICA CENTRAL: REVISÃO E PERSPECTIVAS DESDE EL SALVADOR" de Juan José López Rogel, da Universidad Centroamericana José Siméon Cañas, de El Salvador, que discute a história recente das relações entre a China e a América Central, região, conforme já mencionado, 
historicamente sob forte influência dos Estados Unidos. Apresentando grande quantidade de dados sobre o comércio bilateral entre os países centro-americanos e a China, bem como números sobre investimentos chineses na região, o artigo traz análise detalhada das relações entre a potência asiática e El Salvador, para então discutir os impactos econômicos da pandemia na América Latina em geral e na América Central em particular. Se por um lado a presença chinesa é vista como incômoda por Washington, por outro representa, por meio de ofertas de investimentos e acordos de cooperação, uma alternativa de diversificação das estratégias de desenvolvimento do Istmo Centro-Americano.

\section{Em "COOPERAÇÃO E INTEGRAÇÃO ECONÔMICA INTERNACIONAL} ENTRE CHINA E COLÔMBIA" Alvaro Andrés Escobar-Espinoza e Bernardo Romero-Torres, da Universidad de Cartagena, Colômbia, nos brindam com uma interessante reflexão sobre as relações entre China e Colômbia nestas duas primeiras décadas do século XXI. Diferentemente dos países que viveram a chamada "onda rosa" no período, e que buscaram na China, através do incremento das relações Sul-Sul, uma alternativa para obter maior grau de autonomia no cenário internacional, a Colômbia seguiu como um dos parceiros mais estratégicos dos Estados Unidos na região. Isso no entanto não impediu que o país seguisse a tendência de quase toda a América Latina e o Caribe e incrementasse fortemente relações comerciais com Beijing. Rico em dados e análises, o artigo chama a atenção para a necessidade de diversificação da pauta de exportações da Colômbia para a China, especialmente do setor industrial.

O aprofundamento da globalização ocorrido desde o final do século XX ampliou enormemente as comunicações, barateou os transportes e favoreceu a criação de redes internacionais de imigração. Laura Bogado Bordazar, da Universidad Nacional de La Plata, Argentina, nos apresenta, em “MIGRAÇÃO MODERNA PARA O EXTERIOR? PRINCIPAIS FLUXOS E PROJEÇÕES DA MIGRAÇÃO CHINESA NA REGIÃO SUL-AMERICANA. O CASO DO MERCosul", um amplo levantamento sobre os padrões 
migratórios de chineses em direção à América Latina naquele período, com ênfase no caso dos países do Mercosul. De fato, a abertura chinesa para o mundo, iniciada em 1978, possibilitou mudanças nas políticas migratórias daquele país para outras partes do mundo. Ainda que a ampla maioria dos migrantes chineses tenha se estabelecido em outros países da região Ásia-Pacífico, foram crescentes no período os fluxos migratórios para a América Latina, constituindo redes familiares, redes de clãs e redes internacionais. O artigo aponta, no entanto, que ainda não se observa na região, e em especial nos países do Mercosul, o mesmo padrão de investimentos feitos por estes imigrantes como ocorre na região Ásia-Pacífico.

Maria Francesca Staiano, da Universidad Nacional de La Plata, Argentina, nos propõe, em “O NEO-HUMANISMO CHINÊS: UM NOVO PARADIGMA INTERNACIONAL E JURÍDICO NAS RELAÇÕES INTERNACIONAIS CHINA-AMÉRICA LATINA", refletir sobre a criação em curso de uma nova doutrina de relações humanas e relações entre povos e países a partir da visão chinesa em relação ao planeta e à Humanidade. Valores como solidariedade internacional, relações diplomáticas e econômicas de tipo ganha-ganha e desenvolvimento humano, assim como Direitos Humanos e desenvolvimento sustentável. Itens sem sombra de dúvidas centrais para a construção de uma nova ordem mundial, que seja estruturada sob relações de cooperação e que evite a eclosão de conflitos, sejam de tipo Guerra Fria ou guerra convencional. Cabe aos nossos países, no entanto, avaliar em que medida é possível transformar nossas relações com o gigante asiático de modo a não aprofundar relações econômicas que atentem contra nossos recursos naturais, como tem sido nas relações da nossa região com as grandes potências mundiais desde o período colonial.

\section{Em "INTEGRAÇÃO ENERGÉtICA NA AMÉRICA DO SUL:} PERSPECTIVAS, IMPASSES E OBSTÁCULO" Igor Fuser e Rafael Almeida Ferreira Abrão, da Universidade Federal do ABC, Brasil, nos convidam a 
pensar sobre um tema central para a integração latino-americana mas tantas vezes relegados a segundo plano por muitos governos da região: a questão energética. Os autores mostram que historicamente as iniciativas naquela direção têm se caracterizado por interconexões físicas para o transporte de eletricidade e gás natural, num debate muitas vezes limitado às questões da segurança jurídica dos investidores, sem coordenação regional e distantes da concepção de sustentabilidade. O aprofundamento das relações com a China implica, além do comércio, na recepção de investimentos em infraestrutura de energia e estes, a depender de como forem geridos, podem ser uma oportunidade, segundo os autores, de induzir à cooperação regional.

Como as leitoras e os leitores já estão habituados, Brazilian Journal of Latin American Studies (BJLAS) tem publicado, em todas as suas edições, resenhas críticas de obras recentes produzidas por importantes autores do pensamento social latino-americano e caribenho. Neste número 37 apresentamos "UM OLHAR LATINO-AMERICANO SOBRE A ERA TRUMP", de Ricardo Neves Streich, de Universidade de São Paulo, Brasil. Nesta resenha do livro "Geopolítica y Economía Mundial: El ascenso de China, la era Trump y América Latina", de autoria de Gabriel Merino e Patrício Narodowski, publicado 2019 pela Editora da Universidad Nacional de La Plata, Streich destaca a rica análise feita pelos autores a partir da ascensão econômica e política da China no cenário mundial contemporâneo e a resposta nacionalista produzida nos Estados Unidos com a chegada de Donald Trump à Casa Branca. Streich destaca que o livro refuta as análises de cunho liberal sobre o fenômeno e recupera a perspectiva estruturalista tão tradicional nas Ciências Sociais latino-americanas, como os conceitos de "relações centro-periferia", "dependência" e "hegemonia".

A iniciativa de editar este dossiê surgiu das discussões do Grupo de Trabalho "China y el Mapa del Poder Mundial", do Conselho Latino-Americano de Ciências Sociais (CLACSO). Criado em 2019 o grupo é composto por mais de 30 pesquisadores de 16 diferentes países e tem se 
dedicado a pesquisar e analisar a ascensão da China no cenário mundial e suas implicações para a América Latina e o Caribe.

\section{Carta a las lectoras y los lectores,}

La Brazilian Journal of Latin American Studies (BJLAS) se complace en publicar el número 37 de la revista especializada en estudios sobre América Latina y el Caribe. En esta ocasión, presentamos el dossier “Relaciones China - América Latina y el Caribe", en el que especialistas presentan análisis sobre un tema tan amplio desde diferentes puntos de vista. El trasfondo de las discusiones son las transformaciones que han tenido lugar en las relaciones económicas y de poder mundiales en las últimas décadas. El antiguo orden mundial del posguerra - bajo el liderazgo de Estados Unidos en asociación con Europa Occidental y Japón, y respaldado por la arquitectura de gobernanza global compuesta por las Naciones Unidas, el Fondo Monetario Internacional y el Banco Mundial - ya no refleja el mundo de hoy. Nuevos actores entraron en escena a principios de este siglo. India ha emergido como una potencia económica, Rusia ha recuperado importancia en la arena política mundial después de la débâcle soviética y China, el imperio más importante de la Antigüedad, está en un movimiento acelerado de (re)ascensión al tope del poder mundial.

América Latina y el Caribe, aunque sin un rol de protagonismo global, parecen ser una región fundamental en este período de transición entre el breve orden unipolar estadounidense, que existió entre el fin de la antigua URSS y principios del siglo XXI, y el nuevo orden multipolar en construcción. Mientras que Estados Unidos ha visto a América Latina y el Caribe, desde principios del siglo XIX, como un área natural para su expansión económica, geopolítica y cultural, China tiene en la región un 
proveedor de commodities agrícolas y energéticas fundamentales para su economía. La profundización de los lazos económicos de Pekín con nuestros países, a través del incremento del comercio bilateral, inversiones y préstamos, ha sido una de las principales razones del aumento de las tensiones entre Estados Unidos y China. Las relaciones económicas con la potencia asiática, por un lado, nos dan acceso para exportar nuestros productos a un mercado gigantesco, garantizando un mayor grado de autonomía para nuestros países en las relaciones con los más importantes centros del poder mundial pero, por otro lado, contribuyen a que se mantenga nuestro perfil de naciones exportadoras de productos primarios. Resulta que se trata de relaciones llenas de complejidad, y reflexionar sobre el lugar de nuestra región en esta disputa global por la hegemonía es una tarea fundamental para todas y todos los que estén dispuestos a pensar la realidad contemporánea de América Latina y el Caribe.

Este dossier comienza con el artículo "DE LA GRAN CRISIS A LA GRAN DEPRESIÓN DE LOS 2020's. DESENVOLVIMIENTO DE CHINA, REFLEXIÓN POSTKEYNESIANA Y PERSPECTIVAS", de Alicia Girón, de la Universidad Nacional Autónoma de México, México, que analiza la crisis económica mundial de 2020 provocada por la pandemia del Covid-19 y sus consecuencias en la economía china. La autora señala los graves antecedentes de esta crisis presentes en las medidas económicas ortodoxas implantadas en varios países del mundo por ocasión de la crisis de 2008, con fuertes impactos sobre la actividad productiva y el nivel de empleo. Sin embargo, ella también señala que el Banco Central de China apostó, al mismo tiempo, por una política económica expansiva, como ya hacía desde la admisión del país en la Organización Mundial del Comercio (OMC) en 2001. Más que eso, Pekín apostó, a partir de 2013, en un modelo de desarrollo al exterior, la Nueva Ruta de la Seda, que involucra a Asia, África y América Latina a través de circuitos terrestres y marítimos orientados a integrar proveedores y consumidores de productos vinculados a la economía china. Rico en datos cuantitativos, el artículo 
apunta a posibles consecuencias, en estas alianzas, resultantes de la actual desaceleración de las economías de los países involucrados.

El segundo artículo es de Gabriel Merino, de la Universidad Nacional de La Plata, Argentina, titulado "EL ASCENSO DE CHINA Y LAS DISPUTAS ESTRATÉGICAS EN LOS GRUPOS DOMINANTES DE LOS ESTADOS UNIDOS". En él, el autor presenta a los lectores una profunda discusión sobre las grandes transformaciones de la economía y la geopolítica contemporáneas que involucran a Estados Unidos y China. El enfoque de Merino está en las diversas estrategias desarrolladas por Washington para enfrentar el crecimiento chino y la disputa resultante por la hegemonía global. Al mismo tiempo, el artículo reflexiona sobre las respuestas de Pekín al giro nacionalista-americanista que representó el ascenso al poder de Donald Trump. El autor sostiene que las iniciativas de la Casa Blanca desde entonces han amplificado la crisis del orden mundial y pueden, por eso, favorecer indirectamente a China. Mientras las fuerzas globalistas se debilitan en el Occidente, China está aprovechando la oportunidad para aumentar su influencia estratégica hacia su entorno inmediato, en Asia, y también hacia África y América Latina.

\section{En "EL ATERRIZAJE DEL DRAGÓN EN AMÉRICA DEL SUR: UNA} ANÁLISIS DE LA GEOECONOMÍA, DE LOS CHINA'S POLICY PAPER ON LATIN AMERICA AND THE CARIBBEAN Y DE LA NUEVA RUTA DE LA

SEDA" Bernardo Rodrigues, de la Universidad Federal de Río de Janeiro, Brasil, presenta datos importantes sobre la creciente incorporación de América del Sur en la estrategia de expansión geoeconómica global de China. Analizando el período 2001-2016 a partir de datos cuantitativos y documentos de la política china para la región, el autor demuestra cómo Pekín tiene América Latina y el Caribe como una prioridad para la construcción de un nuevo orden mundial. Esta sería guiada por el multilateralismo, a través de la cooperación entre naciones y la integración de los países del Sur en cadenas globales de valor. Para eso, sostiene Rodrigues, es fundamental que los líderes políticos sudamericanos 
interactúen con Pekín de manera autónoma y coordinada, con el objetivo de garantizar el acceso a tecnologías y conocimientos que permitan establecer relaciones de independencia simétricas en la región en su relación con la potencia asiática.

Lourdes Regueiro Bello, del Centro de Investigaciones de Política Internacional, de Cuba, analiza, en "CENTROAMÉRICA EN LA DISPUTA GEOPOLÍTICA ENTRE CHINA Y ESTADOS UNIDOS" la posición de Centroamérica en la disputa comercial global entre Estados Unidos y China. Como sabemos, este enfrentamiento va más allá de la economía y también tiene implicaciones geopolíticas. Y Centroamérica, por su condición ístmica y bioceánica, tiene un papel estratégico en esta disputa. Washington considera la presencia china en una región tan cercana a su territorio como una amenaza, mientras ejerce una posición similar en relación con Taiwán y gran parte del Mar de China Meridional. La percepción de amenaza va más allá, debido a la invitación de Pekín a los países latinoamericanos para hacer la ya mencionada Nueva Ruta de la Seda. En el caso específico de las naciones centroamericanas, la oferta de recursos económicos que hace Pekín es, por un lado, tentadora y casi irrefutable, pero por otro, puede resultar en duras sanciones por parte de Washington. El artículo de Regueiro nos ayuda a especular sobre los próximos capítulos de este interesante ajedrez.

\section{A continuación, presentamos el artículo "RELACIONES ENTRE} CHINA Y CENTROAMÉRICA: REVISIÓN Y PERSPECTIVAS DESDE EL SALVADOR", de Juan José López Rogel, de la Universidad Centroamericana José Simeón Cañas, de El Salvador, en el que se analiza la historia reciente de las relaciones entre China y Centroamérica, región, como ya se mencionó, históricamente bajo la fuerte influencia de Estados Unidos. Al utilizar una gran cantidad de datos sobre el comercio bilateral entre los países centroamericanos y China, así como cifras sobre las inversiones chinas en la región, el artículo presenta análisis detallado de las relaciones entre la potencia asiática y El Salvador, para luego discutir los 
impactos económicos de la pandemia en América Latina en general y en América Central en particular. Por un lado, la presencia china es vista como incómoda por Washington, al mismo tiempo que, por otro, a través de ofertas de inversión y acuerdos de cooperación, representa una alternativa para diversificar las estrategias de desarrollo del Istmo Centroamericano.

\section{En "COOPERACIÓN E INTEGRACIÓN ECONÓMICA INTERNACIONAL}

ENTRE CHINA Y COLOMBIA" Álvaro Andrés Escobar Espinoza y Bernardo Romero Torres, de la Universidad de Cartagena, Colombia, nos ofrecen una interesante reflexión sobre las relaciones entre China y Colombia en estas dos primeras décadas del siglo XXI. A diferencia de los países que vivieron la Ilamada "ola rosa" en el período, y que buscaron en China, por medio del aumento de las relaciones Sur-Sur, una alternativa para obtener un mayor grado de autonomía en el escenario internacional, Colombia permaneció como uno de los socios más estratégicos de los Estados Unidos en la región. Sin embargo, esto no impidió que el país siguiera la tendencia de casi toda América Latina y el Caribe y aumentase de manera significativa las relaciones comerciales con Pekín. Rico en datos y análisis, el artículo llama la atención sobre la necesidad de diversificar la canasta exportadora de Colombia a China, especialmente el sector industrial.

La profundización de la globalización que se ha producido desde finales del siglo $X X$ ha ampliado enormemente las comunicaciones, abaratado los costos del transporte y favorecido la creación de redes de inmigración internacional. Laura Bogado Bordazar, de la Universidad Nacional de La Plata, Argentina, nos presenta, en "¿MIGRACIÓN DE ULTRAMAR MODERNA? PRINCIPALES FLUJOS Y PROYECCIONES DE LA MIGRACIÓN CHINA EN LA REGIÓN SUDAMERICANA. EL CASO DEL MERCOSUR" una amplia mirada sobre los patrones migratorios chinos hacia América Latina en ese período, con énfasis en el caso de los países del Mercosur. De hecho, la apertura de China al mundo, que comenzó en 1978, permitió cambios en las políticas de migración de ese país a otras partes del mundo. Aunque la gran mayoría de los migrantes chinos se 
establecieron en naciones de la región de Asia-Pacífico, los flujos migratorios hacia América Latina aumentaron durante el período, constituyendo redes familiares, redes de clanes y redes internacionales. El artículo señala, sin embargo, que aún no se observa en la región, y especialmente en los países del Mercosur, el mismo patrón de inversiones que realizan estos inmigrantes, como ocurre en la región Asia-Pacífico.

Maria Francesca Staiano, de la Universidad Nacional de La Plata, Argentina, nos propone, en "EL NEO-HUMANISMO CHINO: UN NUEVO PARADIGMA JURÍDICO E INTERNACIONAL EN LAS RELACIONES INTERNACIONALES CHINA-AMÉRICA LATINA", reflexionar sobre la creación, hoy, de una nueva doctrina de relaciones humanas y relaciones entre pueblos y países desde la visión china del planeta y de la humanidad. Valores como la solidaridad internacional, las relaciones diplomáticas y económicas de beneficio mutuo y el desarrollo humano, así como los derechos humanos y el desarrollo sostenible. Indudablemente, elementos centrales para la construcción de un nuevo orden mundial, que se estructura bajo la cooperación y que evita el estallido de conflictos, sean de tipo Guerra Fría o guerra convencional. Depende de nuestros países, sin embargo, evaluar en qué medida es posible transformar nuestras relaciones con el gigante asiático para no profundizar amenazas a nuestros recursos naturales, como ha sido el caso de las relaciones de nuestra región con las grandes potencias mundiales desde el período colonial.

\section{En "INTEGRACIÓN ENERGÉTICA EN SUDAMÉRICA: PERSPECTIVAS,}

IMPASES Y OBSTÁCULOS" Igor Fuser y Rafael Almeida Ferreira Abrão, de la Universidade Federal do ABC, Brasil, nos invitan a pensar en un tema central de la integración latinoamericana que por tantas veces es relegado a un segundo plan por muchos gobiernos de la región: el tema energético. Los autores muestran que históricamente las iniciativas en esa dirección se han caracterizado por interconexiones físicas para el transporte de electricidad y gas natural, en un debate que muchas veces se limita a temas de seguridad jurídica para los inversionistas, sin coordinación entre 
los gobiernos de la región y distante del concepto de sustentabilidad. La profundización de las relaciones con China implica, además del comercio, la recepción de inversiones en infraestructura energética y estas, dependiendo de cómo se gestionen, pueden ser una oportunidad, según los autores, para inducir la cooperación regional.

Como es de costumbre Brazilian Journal of Latin American Studies (BJLAS) presenta reseñas críticas de obras recientes publicadas por destacados y destacadas pensadores de la realidad latinoamericana y caribeña. En esta oportunidad contamos con "UNA MIRADA

LATINOAMERICANA A LA ERA TRUMP", de Ricardo Neves Streich, de la Universidade de São Paulo, Brasil. En esta reseña del libro "Geopolítica y Economía Mundial: El ascenso de China, la Era Trump y América Latina", de Gabriel Merino y Patrício Narodowski, publicado en 2019 por la Editorial de la Universidad Nacional de La Plata, Streich destaca el rico análisis realizado por los autores sobre el ascenso económico y político de China en el escenario mundial contemporáneo y la respuesta nacionalista producida desde Estados Unidos con la llegada de Donald Trump a la Casa Blanca. Streich señala que el libro refuta los análisis liberales del fenómeno y recupera la perspectiva estructuralista tan tradicional en las ciencias sociales latinoamericanas, como los conceptos de "relaciones centro-periferia", "dependencia" y "hegemonía”.

La iniciativa de editar este dossier es fruto de las discusiones del Grupo de Trabajo "China y el Mapa del Poder Mundial", del Consejo Latinoamericano de Ciencias Sociales (CLACSO). Creado en 2019, el grupo está compuesto por más de 30 investigadores de 16 distintos países y se ha dedicado a investigar y analizar el ascenso de China en el escenario mundial y sus implicaciones para América Latina y el Caribe. 


\section{Letter to the readers,}

The Brazilian Journal of Latin American Studies (BJLAS) is pleased to publish its issue 37. BJLAS is a scientific journal specialized in Latin America and the Caribbean studies. On this occasion, we present the dossier "China - Latin America and the Caribbean Relations", in which experts on the subject analyze such a vast subject from different points of view. The background to the discussions are the transformations that have taken place in global economic and geopolitics in recent decades. The old post-war world order - led by the United States in partnership with Western Europe and Japan and supported by the global governance architecture composed by the United Nations, the International Monetary Fund and the World Bank - no longer reflects the contemporary international order. New actors entered the scene at the beginning of this century. India has emerged as an economic power, Russia has regained relevance in the world political arena after the Soviet débâcle and China, the greatest empire of the Old Age, is now in an accelerated movement of (re) ascension to the top of the world arena.

Latin America and the Caribbean, although without a strategic global role, seem to be a fundamental region in this period of transition between the brief US unipolar order, which existed between the end of the former USSR and the beginning of the 21st century, and the new order multipolar under construction. While the United States has seen Latin America and the Caribbean, since the beginning of the 19th century, as a natural area for its economic, geopolitical and cultural expansion, China has in the region a supplier of agricultural and energy commodities fundamental to its economy. The deepening of Beijing's economic ties with our countries, through the increase of bilateral trade, investments and loans, has been one of the main reasons behind the rising tensions between the United States and China. Economic relations with Beijing give 
to Latin America and the Caribbean access to export our products to a gigantic market, guaranteeing a relative level of autonomy for our countries in their relations with the most powerful countries of the world. But it contributes, however, to reinforce our primary exporting profile. Thus, it is important to point out that these are relationships full of complexity, and reflecting on the role of our region in this global dispute for hegemony. It is a fundamental task for all and everyone who is willing to think about the contemporary Latin America and the Caribbean.

This dossier begins with the article "FROM THE GREAT CRISIS TO THE GREAT DEPRESSION OF THE 2020's. CHINA'S DEVELOPMENT, POST-KEYNESIAN REFLECTION AND PERSPECTIVES" by Alicia Girón, of the Universidad Nacional Autónoma de México, Mexico. It discusses the world economic crisis of 2020 caused by the Covid-19 pandemic and its consequences on the Chinese economy. The author points out the antecedents of this crisis present in the orthodox economic policies implanted in several countries of the world due to the crisis of 2008, with strong impacts on the industry sector and the level of employment. However, she also observes that the Chinese Central Bank bet, in those same years, on an expansive economic policy, as it had been doing since the country was admitted to the World Trade Organization (WTO), in 2001. More than that, Beijing has been strengthening, since 2013, in a development model outward to invest in foreign countries, the New Silk Road, involving Asia, Africa and Latin America through terrestrial and maritime circuits aimed at integrating suppliers and customers of products linked to the Chinese economy. Rich in quantitative data, the article points to the possible consequences, in these partnerships, resulting from the current slowdown caused by the pandemic in the economies of the countries involved.

The second article is "THE RISE OF CHINA AND THE STRATEGIC DISPUTES IN THE DOMINANT GROUPS OF THE UNITED STATES" written by Gabriel Merino, from Universidad Nacional de La Plata, Argentina. He 
presents a detailed discussion about the main changes of contemporary economics and geopolitics involving the United States and China. Merino's focus is on the various strategies developed by Washington to face Chinese growth and the resulting dispute for the global hegemony. At the same time, the article reflects on Beijing's responses to the nationalist-Americanist spin represented by Donald Trump's rise to the power. The author asserts that the White House's initiatives since then have amplified the crisis of the world order and may, because of this, indirectly favor China. While globalist forces are weakening in the Western world, China is taking the opportunity to expand its strategic influence towards Asia, and also Africa and Latin America.

In "THE DRAGON'S LANDING IN SOUTH AMERICA: AN ANALYSIS OF GEOECONOMICS, CHINA'S POLICY PAPER ON LATIN AMERICA AND THE CARIBBEAN AND THE NEW SILK ROAD" Bernardo Rodrigues, from the Universidade Federal do Rio de Janeiro, Brazil, presents important data on the increasing incorporation of South America in China's global geoeconomic expansion strategy. Analyzing the period 2001-2016 based on quantitative data and Chinese policy papers for the region, the author demonstrates how Beijing considers Latin America and the Caribbean a priority to the construction of a new world order. This would be guided by multilateralism, through cooperation between nations and integration of the countries of poor and developing countries into global value chains. To this purpose, the article argues, it is essential that South American political leaders interact with Beijing in an autonomous way, to ensure access to technologies and knowledge that allow the establishment of symmetrical independence relations in the region in relation to China.

Lourdes Regueiro Bello, from the Centro de Investigaciones de Política Internacional, of Cuba, discusses, in "CENTRAL AMERICA IN THE GEOPOLITICAL DISPUTE BETWEEN CHINA AND THE UNITED STATES", the position of Central America in the global trade dispute between the United States and China. As we know, this confrontation goes beyond the 
economy, and also has geopolitical implications. And Central America, due to its isthmic and bioceanic condition, has a strategic role in this dispute. Washington considers the Chinese presence in an area so close to its territory as a threat while plays a similar position in relation to Taiwan and a part of the South China Sea. The perception of threat goes further because of Beijing's invitation to Latin American countries to participate in the already mentioned New Silk Road. In the specific case of Central American nations, the offer of economic resources made by China is, on the one hand, tempting and almost irrefutable, but on the other hand it may result in harsh sanctions by Washington. Regueiro's article helps us to speculate on the upcoming chapters of this interesting chess.

The following article, "RELATIONS BETWEEN CHINA AND CENTRAL AMERICA: REVISION AND PERSPECTIVES FROM EL SALVADOR", by Juan José López Rogel, from Universidad Centroamericana José Simeón Cañas, El Salvador, discusses the recent history of relations between China and Central America. It is a region, as already mentioned, historically under strong influence from the United States. Presenting in a through comparative perspective a large amount of data on bilateral trade between Central American countries and China, as well as Chinese investments in the region, the article provides a detailed analysis of the relations between the Asian country and El Salvador, to then discuss the economic impacts of pandemic in Latin America in general and Central America in particular. On the one hand, the Chinese presence is seen as uncomfortable by Washington, but on the other hand, through investment offers and cooperation agreements, it represents an alternative to diversify the development strategies of the Central American Isthmus.

In "COOPERATION AND INTERNATIONAL ECONOMIC INTEGRATION BETWEEN CHINA AND COLOMBIA" Alvaro Andrés Escobar-Espinoza and Bernardo Romero-Torres, from the Universidad de Cartagena, Colombia, offer us an interesting reflection on the relations between China and Colombia in these first two decades of the 21st century. 
Unlike the countries that experienced the so-called "Pink Tide" in the two frist decades of this century, and that sought in China, through the increase of South-South relations, an alternative to obtain a increasing levels of autonomy in the international scenario, Colombia continued as one of the most strategic partners of the United States in the region. This, however, did not exclude the country from following the trend of almost the entire region to increase economic relations with Beijing. Rich in data and analysis, the article draws attention to the need to diversify the Colombian export basket to China, especially the industrial goods.

The process of deepening globalization that has occurred since the end of the 20th century has expanded communications strongly, made transportation cheaper and favored the creation of international immigration networks. Laura Bogado Bordazar, from the Universidad Nacional de La Plata, Argentina, in "MODERN OVERSEAS MIGRATION? MAIN FLOWS AND PROJECTIONS OF CHINESE MIGRATION IN THE SOUTH AMERICAN REGION. THE CASE OF MERCOSUR" brings us a comprehensive survey on the migratory patterns of Chinese individuals towards Latin America, with an emphasis on the Mercosur countries. In fact, China's reforms, which began in 1978, enabled changes in that country's immigration policies to other parts of the world. Although the vast majority of Chinese migrants settled in other countries in the Asia-Pacific region, migratory flows to Latin America increased in the last decades, constituting family networks, clan networks and international networks. The article adverts, however, that the same pattern of investments made by those immigrants is still not observed in the region, and particularly in the Mercosur countries, as occurs in the Asia-Pacific region.

Maria Francesca Staiano, from Universidad Nacional de La Plata, Argentina, proposes, in "CHINESE NEO-HUMANISM: A NEW INTERNATIONAL AND LEGAL PARADIGM IN CHINA-LATIN AMERICA INTERNATIONAL RELATIONS" to reflect on the ongoing creation of a new 
doctrine of human relations and relations between peoples and countries from the Chinese view of the planet and humanity. Values such as international solidarity, win-win diplomatic and economic relations and human development, as well as human rights and sustainable development. Undoubtedly central items for the construction of a new world order, that will be structured under cooperative relations and avoids the outbreak of conflicts, whether of Cold War type or conventional war type. It is up to our countries, however, to devise relations with China that do not deep economic relations that threaten our natural resources, as has been the pattern of Latin America and the Caribbean with the great world powers since the colonial period.

In "ENERGY INTEGRATION IN SOUTH AMERICA: PERSPECTIVES, DEADLOCKS AND OBSTACLES" Igor Fuser and Rafael Almeida Ferreira Abrão, from the Universidade Federal do ABC, Brazil, bring the readers with a central theme for Latin American integration but so often relegated to the background by many governments of the region: the energy issue. The authors show that historically the initiatives in that direction have been characterized by physical interconnections for the transportation of electricity and natural gas, in a debate that is often limited to issues of legal security for investors, without regional coordination and distant from the idea of sustainable development. The deepening of relations with China implies, in addition to trade, the reception of investments in energy infrastructure and these, depending on how they are managed, can be an opportunity, according to the authors, to induce regional cooperation.

As the reader can notice in the previous issues, the Brazilian Journal of Latin American Studies (BJLAS) also publishes book reviews on recent works or studies by great authors of Latin American and the Caribbean social thought. For the issue 37 we present the book review "A LATIN AMERICAN LOOK AT THE TRUMP ERA", by Ricardo Neves Streich, from the University of São Paulo, Brazil, based on "Geopolitics and World Economy: The rise of China, it was Trump and Latin America", written by 
Gabriel Merino and Patrício Narodowski and published in 2019 by the Universidad Nacional de La Plata. He highlights the rich analysis made by authors from the economic and political rise of China in the contemporary world scene and the nationalist response of the United States under Trump's government. Streich points out that the book refutes liberal analyzes and recovers the structuralist perspective so traditional in the Latin American Social Sciences, such as the concepts of "center-periphery relations", "dependence" and "hegemony".

The initiative to edit this dossier came from the discussions of the Working Group "China and the Map of World Power", of Consejo Latinoamericano de Ciencias Sociales (CLACSO). Created in 2019, the group is composed of more than 30 researchers from 16 different countries and has been dedicated to researching and analyzing the rise of China on the global arena and its implications for the Latin America and the Caribbean region. 